\title{
Kommentar zur Guideline zum Vorgehen bei suspektem und positivem zytologischen Abstrich der Cervix Uteri
}

\author{
R. Rondez, J. Kunz, C. Moll
}

1 Arbeitsgruppe «Guideline Zervixabstrich». Guideline zum Vorgehen bei suspektem und positivem zytologischen Abstrich der Cervix Uteri. Schweiz Ärztezeitung 2003;84(3):82-92.

2 Wright TC Jr, Cox JT, Massad LS, Twiggs, LB, Wilkinson EJ. 2001 Consensus Guidelines for the management of women with cervical cytological abnormalities. JAMA 2002;287:2120-9.

3 Saslow D, Runowicz CD, Solomon D, Moscicki AB Smith RA, Eyre HJ, Cohen C. American Cancer Society Guideline for the Early Detection of Cervical Neoplasia and Cancer. CA Cancer J Clin 2002;52:342-62.

Korrespondenz:

PD Dr. med. R. Rondez Institut für Klinische Pathologie Zytologie und angewandte Zytometrie

Streulistrasse 14

CH-8032 Zürich
Die zum Jahresbeginn erschienene Empfehlung der Kommission Qualitätssicherung der Schweizerischen Gesellschaft für Geburtshilfe und Gynäkologie (SGGG) zur Prävention des Zervixkarzinoms [1] erwartete man mit Spannung, wurden doch in den letzten Jahren gerade auf diesem Gebiet eine Vielzahl neuer Erkenntnisse gewonnen und wichtige Fortschritte erzielt. Ebenso unverkennbar ist ein grosser Informationsbedarf von seiten der Öffentlichkeit wie auch des gynäkologisch tätigen Arztes, der mit einer Flut von Publikationen, Prospekten und ständigen Kontroversen um neue Vorsorgestrategien konfrontiert ist. Umso mehr hätte man von den Experten eine klärende und aktualisierte Stellungnahme erwartet. Präsentiert wird statt dessen eine Guideline, die nach Angaben der Autoren die internationale Fachliteratur nur bis Oktober 2000 berücksichtigt und wichtige Fragen ausklammert. So werden in dem vorliegenden, umfangreichen Dokument die wirklichen, anstehenden Probleme kaum berührt, der Wert der Dünnschichtmethoden und des HPV-Tests in Frage gestellt oder stark relativiert und die bahnbrechenden Fortschritte der Grundlagenforschung mit ihren Implikationen für die Prävention und Therapie des Zervixkarzinoms nicht erwähnt. Angesichts solcher gravierender Mängel einer de facto verbindlichen Guideline scheint es uns dringlich, an dieser Stelle darauf einzugehen. Dabei handelt es sich nicht nur um eine persönliche Meinung, sondern um Schlussfolgerungen, die aus der heute verfügbaren, internationalen Literatur gezogen werden müssen.

\section{Dünnschichtmethoden \\ (Monolayer-Methoden, flüssigkeits- gestützte Präparationsverfahren)}

Entgegen den Angaben der Guideline stehen die Vorteile dieser Methoden, die von der FDA anerkannt sind und die derzeit in der Schweiz für 60 bis $70 \%$ der PAP-Abstriche verwendet werden, nicht mehr zur Diskussion.

\section{HPV-Typisierung \\ (HPV = Humane Papillomaviren)}

Der Begriff ist insofern ungenau, als zumeist Zervixabstriche nur auf onkogene HPV-Typen (sogenannte High-risk-HPV) untersucht werden, die allein von klinischer Bedeutung sind. Als Nachweismethode hat sich vor allem das Hybrid Capture System II (Digene/Abbott) bewährt, mit welchem die DNA der häufigsten High-risk-HPV(HR-HPV-)Typen mit Hilfe eines RNA-Sondengemisches nachgewiesen und mittels Chemolumineszenz quantitativ bestimmt wird. Die Messwerte sind abhängig von der Zahl der erfassten infizierten Zellen («sampling error») wie auch von der Menge der in der Zelle enthaltenen episomalen oder integrierten Virus-DNA. Als Grenzwert verwendet man zur Optimierung von Sensitivität und Spezifität eine HPV-DNA-Menge von $1 \mathrm{pg} / \mathrm{ml}$.

HPV-Tests werden heute nach internationalen Empfehlungen in folgenden Fällen durchgeführt:

- zur Erfassung von Risikopatientinnen bei unklaren zytologischen Befunden (sogenannte ASCUS-Fälle = Atypical squamous cells of undetermined significance). Diese Indikation wird seit 2002 von der American Society of Colposcopy and Cervical Pathology [2] empfohlen;

- in Kombination mit der Dünnschichtzytologie bei über 30 Jahre alten Frauen zur Verlängerung der zytologischen Kontrollintervalle. Diese Indikation, die auf dem hohen negativen Vorhersagewert eines doppelt negativen Testes (Zytologie/HPV-Nachweis) beruht, wird seit 2002 von der American Cancer Society empfohlen [3] und wird derzeit in grossen europäischen Studien auf ihre Kosteneffektivität geprüft. Die Anerkennung durch die FDA steht unmittelbar bevor.

- bei persistierenden Dysplasien;

- bei Risikopatientinnen mit Immunschwächen (AIDS, Organtransplantierte usw.);

- bei Patientinnen mit Kondylomen und Präkanzerosen der Anogenitalregion; 
- zur Therapiekontrolle bzw. zur Früherfassung von Rezidiven nach Konisation.

\section{High-Risk-HPV und Gebärmutterhalskrebs}

Entgegen den relativierenden Ausführungen der Guideline ist heute bekannt, dass eine Progression einer Dysplasie zum Karzinom nur bei persistierendem HR-HPV-Infekt erfolgt [4]. Auch sind die molekularbiologischen Aspekte einer solchen Progression in wichtigen Teilen geklärt, und der kausale Zusammenhang zwischen HRHPV-Infekt und Zervixkarzinom ist über jeden Zweifel erhaben. Statistisch gesehen ist diese Korrelation enger als diejenige zwischen dem Zigarettenrauchen und dem Lungenkrebs. Genitale HPV-Infekte gehören im übrigen zu den am weitesten verbreiteten und sich heute am raschesten ausbreitenden Infektionen. Dies erklärt die in den Industrieländern bei jungen Frauen beobachtete zunehmende Häufigkeit von zervikalen Krebsvorstufen (Carcinoma in situ) und Adenokarzinomen. Letztere sind besonders aggressiv und machen derzeit, je nach Studie und Tumorklassifikation, 20-30\% aller Zervixkarzinome aus.

\section{Aktuelle Probleme und Ausblick}

Vergessen wird oft, dass selbst regelmässige zervikale Vorsorgeuntersuchungen keinen absoluten Schutz vor einem Zervixkarzinom bieten. So hatte in einer englischen Studie mehr als die Hälfte der Frauen, bei denen ein invasives Zervixkarzinom diagnostiziert wurde, regelmässig am nationalen Krebsvorsorgeprogramm teilgenommen [5]. Besondere Probleme bieten die Adenokarzinome, deren Inzidenz und Mortalität nach Einführung der Vorsorgeuntersuchungen nicht zurückgingen. Da die zytologische Methodik an ihre Grenzen stösst, die HPV-Diagnostik aber neue Möglichkeiten eröffnet, muss über eine verbesserte, neue Strategie der Vorsorge diskutiert werden. Offene Fragen bestehen nach wie vor zu den komplexen immunologischen Vorgängen, die für den individuellen Verlauf von HPV-Infekten von entscheidender Bedeutung sind und vielen Einflüssen unterliegen. Auch kann der HPV-Virusbefall bei der gleichen Person zeitabhängig stark variieren, so dass ein einmaliger Test keine Rückschlüsse auf den künftigen
HPV-Status erlaubt. Insbesondere erlaubt er nicht, zwischen den viel häufigeren transienten (etwa 90\%) und den persistierenden Infekten zu unterscheiden, die allein von klinischer Bedeutung sind.

Fortschritte zu obigen Fragen sind zu erwarten

- durch die Bereitstellung geeigneter serologischer Marker;

- durch eine verfeinerte HPV-Diagnostik (HPVQuantifizierung, Berücksichtigung der Virusintegration, der Expression der viralen Onkoproteine E6 und E7 usw.);

- durch direkten Nachweis HR-HPV-infizierter, dysplastischer oder neoplastischer Zellen am zytologischen Präparat mittels In-situHybridisierung oder immunhistochemischer Methoden. Entsprechende Versuche mit Hilfe des immunhistochemischen Nachweises von p16INK4a-Protein sind erfolgversprechend.

Mit Sicherheit ist zu erwarten, dass die Bedeutung der HPV-Diagnostik weiter zunimmt und sprunghaft ansteigt, wenn typenspezifische Impfstoffe für die Prävention und Therapie des Zervixkarzinoms zur Verfügung stehen.

\section{Schlussfolgerungen}

Zusammenfassend ist festzustellen, dass die neuen Technologien jetzt schon eine entscheidende Verbesserung der zervikalen Krebsvorsorge darstellen, deren Problematik ganz von der überragenden Bedeutung der HPV-Infekte bestimmt wird. Sachkundig eingesetzt, erlauben diese Methoden nicht nur, das Krebsrisiko zu senken, sondern auch beträchtliche Kosten einzusparen, indem dank grösserer Effizienz unnötige klinische, zytologische und histologische Kontrollen vermieden werden können. Die freiwerdenden Ressourcen können unter anderem genutzt werden, betroffene Patientinnen ausreichend über die wahren Risiken und Heilungschancen einer HPV-Infektion aufzuklären.

Aufgrund des raschen Wissenszuwachses und der Brisanz des Themas auch in der Öffentlichkeit wäre es im Interesse der Gynäkologen, Onkologen und der gesamten Ärzteschaft, die Guideline baldmöglichst in einer dem heutigen Stand des Wissens entsprechenden Fassung erneut zu publizieren. 